\title{
Methicillin Resistant and Biofilm Producing Staphylococcus species Isolated from Different Clinical Specimens and Antibiotic Susceptibility Pattern of Isolates
}

\author{
Pawana Pandey ${ }^{1}$, Anup Bastola ${ }^{2}$, Beena Shrestha ${ }^{1}$, Puspa Raj Dahal ${ }^{1}$, Pradeep Kumar Shah ${ }^{1}$ \\ ${ }^{1}$ Department of Microbiology, Tri-Chandra Multiple Campus, Tribhuvan University, Kathamandu, Nepal \\ 2Sukraraj Tropical and Infectious Disease Hospital, Kathmandu, Nepal
}

Corresponding author: Pradeep Kumar Shah; Department of Microbiology, Tri-chandra Multiple Campus, Tribhuvan University, Kathmandu, Nepal, Email: pkshah210@gmail.com

\begin{abstract}
Objectives: To determine prevalence of Methicillin Resistant Staphylococcus aureus in different clinical specimens and biofilm production along with antimicrobial susceptibility pattern of isolates.

Methods: Cross-sectional study was conducted from September 2019 to February 2020at Sukraraj Tropical and Infectious Disease Hospital. Total 3091 clinical specimens like blood, urine, sputum, pus, swab, body fluid were processed. Identification was done on the basis of colony characteristics, gram staining, culture in Mannitol Salt Agar, coagulase and oxidation fermentation test. Antibiotic susceptibility test and biofilm detection were performed by Kirby Bauer's disc diffusion methods and Tissue Culture Plate technique (TCP) respectively. Methicillin resistant Staphylococcus species were detected by using Cefoxitin disc.
\end{abstract}

Results: Out of 52 Staphylococcus species, 39 were Staphylococcus aureus and 13 were Coagulase negative Staphylococcus species. Highest numbers of Staphylococcus species were isolated from blood Sixteen (30.8\%) were Methicillin resistant Staphylococcus aureus (MRSA) and 5(9.6\%) were Methicillin resistant Coagulase negative Staphylococcus species. There was no significant association $(\mathrm{p}=0.25)$ between age group and prevalence of MRSA, MSSA, MRCoNS and MSCoNS. Methicillin resistant Staphylococcus species were resistant to antibiotics like amoxicillin, cloxacillin, erythromycin and higher sensitivity was found in gentamycin. Among 52 Staphylococcal isolates, 11(21.1\%) were biofilm producers and $41(78.9 \%)$ were non biofilm producers. $90.9 \%$ of $90.9 \%$ of Biofilm producing Staphylococcus species were resistant towards penicillin and erythromycin

Conclusion: The study shows Methicillin resistant Staphylococcus species were resistant to most antibiotics and rate of resistance was slightly higher in biofilm producing isolates comparing to other isolates. resistance. Regular surveillance of methicillin resistance Staphylococcus species and routine screening of biofilm production is important.

Keywords: Staphylococcus species, TCP, MRSA, Biofilm, Antibiotic susceptibility.

\section{INTRODUCTION}

Staphylococcus species, gram positive cocci, are common causes of human infections like wound infections, septicemia and toxic shock syndrome. They are responsible for variety of diseases like infection of heart (endocarditic), infection of bone (osteomyelitis), central nervous system infections such as brain abscesses \&

Date of Submission: August 28, 2020

Published Online: December, 2020 pneumonia. Depending on the strains and the site of infection, they can cause invasive infections and/or toxin-mediated diseases. The pathophysiology varies greatly depending on the type of $S$. aureus infection. Different mechanisms for evasion of the host immune response include the production of an antiphagocytic capsule, sequestering of host antibodies or antigen

Date of Acceptance: October 22, 2020

DOI: https:/ / doi.org/10.3126/tujm.v7i0.33796 
masking by Protein A, biofilm formation, intracellular survival, and blocking chemotaxis of leukocytes.

Biofilm is an assemblage of microbial cells irreversibly with a surface and enclosed in a matrix of primarily polysaccharide material (Donlan 2002). Biofilm formation is recognized method to establish and maintain infections and increase its persistence and boosts level of antimicrobial resistance.Biofilm are associated with many medical conditions like indwelling medical devices, dental plaque, upper respiratory tract infections, peritonitis, and uro-genital infections (Reid G 1999). Important character of biofilm is their increased tolerance to the antimicrobial agents (Wimpenny et al. 2000). Resistance may be due to delayed penetration of antimicrobial agent, altered growth rate of biofilm and other physiological changes (Donlan and Costerton 2002).

With the current emergence of antimicrobials resistance MRSA has been able to evolve rapidly and create new clinical problems. MRSA has ability to survive in the presence of penicillin-like antibiotics, which normally prevent bacterial growth by inhibiting synthesis of cell wall material. MRSA mediates through an altered protein called low affinity penicillin binding protein (PBP2a). PBP2a is encoded by mecA gene and is present in chromosomal mobile genetic element called Staphylococcal cassette chromosome mec (SCCmec). mecA gene is a resistance gene which stops $\beta$-lactam antibiotics from inactivating the enzymes (transpeptidases) critical for cell wall synthesis. In fact, many strains of MRSA exhibit resistant to both $\beta$-lactams and aminoglycosides.

Production of biofilms can be a marker of virulence (Jain and Agarwal 2009) and MRSA biofilms becomes resistant to almost all available antimicrobial agents used for its treatments (Gotz 2002). MRSA and biofilm producing MRSA are becoming more resistant towards almost all available antimicrobial agents commonly methicillin, ampicillin, Ofloxacin, tetracycline, ciprofloxacin, cotrimoxazole, etc. Despite the development of antimicrobial therapy Methicillin resistance Staphylococcus species are recognized as a major cause of nosocomial infection resulting in significant morbidity and mortality.

\section{MATERIALS AND METHODS}

This cross sectional hospital based study was carried out at Sukraraj Tropical Infectious Disease Hospital,
Kathmandu where data collection, identification of Staphylococcus species, antimicrobial susceptibility test and detection of Methicillin resistant Staphylococcus species were done and detection of biofilm formation was done in Med-Micro Research Laboratory Babarmahal from September 2019 to February 2020. A total of 3091 clinical samples including blood, urine, sputum, pus/wound swab, throat swab, body fluid were collected from outpatient suspected of different infections during this period.

Isolation and identification: The received specimens were immediately cultured in Blood Agar (BA), MacConkey Agar (MA), Chocolate Agar(CA) and also Cysteine lactose and electrolyte deficient agar (CLED) was used for urine sample. Suspected $S$. aureus colonies were then inoculated onto Mannitol Salt Agar and incubated.Identification of Staphylococcus species was done on the basis of colony characteristics, gram staining, culture in Mannitol Salt Agar (MSA), and coagulase and oxidation fermentation test.

Antibiotic susceptibility test and confirmation of Methicillin resistant Staphylococcus species: All Staphylococcus species isolates were subjected to in-vitro antimicrobial susceptibility test by Kirby-Bauer disc diffusion method using Mueller Hinton Agar (MHA) as recommended by Clinical laboratory Standard Institute. Commercially available antibiotic tested from HiMedia Company were amoxicillin $(10 \mathrm{mcg})$, cefoxitin (30mcg), cefixime (5mcg), ciprofloxacin (5mcg), cotrimoxazole $(25 \mathrm{mcg})$, coxacillin $(5 \mathrm{mcg})$, clindamycin (2mcg), erythromycin (15mcg), gentamycin (10mcg), nitrofurantion (300 $\mathrm{mcg})$, penicillin (10 mcg), and tetracycline (30mcg).

Conformation of MRSA and Methicillin Resistant Coagulase negative Staphylococcus species MRCoNS was done by using cefoxitin $(30 \mathrm{mcg})$. Diameter of zone of inhibition $\leq 21 \mathrm{~mm}$ was considered as methicillin resistant whereas diameter $\geq 22 \mathrm{~mm}$ was considered as methicillin sensitive (CLSI 2019).

Preservation of isolates and screening of biofilm production in Staphylococcus species: Isolates were preserved in Tryptic Soya Broth with $20 \%$ glycerol in eppendorf tube and kept at $-70^{\circ} \mathrm{C}$ until subsequent tests and same eppendorf tube was transported to laboratory with ice pack for detection of biofilm formation. Biofilm formation was detecetd by Tissue Culture Plate Technique. Isolates from eppendorf tube was then sub 
cultured in NA or MHA. Organisms isolated from fresh agar plates were inoculated in $10 \mathrm{~mL}$ of Trypticase soy broth (TSB) supplemented with $1 \%$ glucose and incubated at $37^{\circ} \mathrm{C}$ for $24 \mathrm{hrs}$. The cultures were then diluted 1:100 with fresh medium. Individual wells of sterile 96 well flat bottom polystyrene tissue culture treated plates were filled with $200 \mu \mathrm{L}$ of the diluted cultures. The control organisms were also incubated, diluted and added to tissue culture plate. Negative control wells contained TSB with $1 \%$ glucose. The plates were incubated at $37^{\circ} \mathrm{C}$ for $24 \mathrm{~h}$. After incubation, contents of each well were removed by gentle tapping. The wells were washed with $0.2 \mathrm{~mL}$ of phosphate buffer saline ( $\mathrm{pH} 7.2$ ) four times to remove free-floating bacteria. Biofilm formed by bacteria adherent to the wells were fixed by $2 \%$ sodium acetate and then stained by crystal violet $(0.1 \%)$. Excess stain was removed by using deionized water and plates were kept for drying. Optical density (OD) of stained adherent biofilm was obtained by using micro ELISA autoreader (model 680, Biorad, UK) at wavelength $570 \mathrm{~nm}$. The experiment was performed in triplicate and repeated three times (Hassan et al. 2011). The interpretation of biofilm production was done according to the criteria of Stepanovic et al. (2007).
Average OD value Biofilm formation $\leq \mathrm{ODc} / \mathrm{ODc}<\sim \leq 2 \mathrm{x}$ ODc Non/ Weak $2 x O D c<\sim \leq 4 x O D c \quad$ Moderate

$>4 \times$ ODc Strong

Optical density cut-off value (ODc) = average OD of negative control $+3 x$ standard deviation (SD) of negative control.

Data analysis: Data analysis was done using computer based software program Statistical Package For The Social Sciences SPSS version 21 and p-value was calculated by using Chi Square test

\section{RESULTS}

Among 3091 clinical samples, 239 showed culture positive with $60(25.11 \%)$ gram positive bacteria. Out of 60 gram positive bacteria, $52(86.67 \%)$ were Staphylococcus species with 39 (65\%) Staphylococcus aureus and 13 (21.67\%) Coagulase negative Staphylococcus species (CoNS).

Out of 52 Staphylococcus species prevalence of MRSA was $16(30.8 \%)$, and MRCoNS was 5(9.6\%). Highest number was obtained from blood. There was no significant association $(p=0.98)$ and $(p=0.29)$ between type of sample and prevalence of MRSA and MSSA and MRCoNS and MSCoNS respectively (Table 1).

Table 1: Prevalence of MRSA, MSSA, MRCoNS and MSCoNS in different clinical specimens

\begin{tabular}{lccccccc}
\hline \multicolumn{1}{c}{ Samples } & MRSA & MSSA & P-value & MRCoNS & MSCoNS & P-value & Total \\
\hline Blood & $9(27.8 \%)$ & $14(42.4 \%)$ & & $5(15.2 \%)$ & $5(15.2 \%)$ & & $33(63.5 \%)$ \\
Urine & $1(50 \%)$ & $1(50 \%)$ & & - & - & $2(3.8 \%)$ \\
Sputum & $4(36.4 \%)$ & $5(45.5 \%)$ & 0.98 & - & $2(18.1 \%)$ & 0.29 & $11(21.1 \%)$ \\
Pus & $2(40 \%)$ & $3(60 \%)$ & & - & $1(20 \%)$ & $5(9.6 \%)$ \\
\hline Totals & $\mathbf{1 6 ( 3 0 . 8 \% )}$ & $\mathbf{2 3 ( 4 4 . 2 \% )}$ & & $\mathbf{5 ( 9 . 6 \% )}$ & $\mathbf{8 ( 1 5 . 4 \% )}$ & $\mathbf{5 2 ( 1 0 0 \% )}$ \\
\hline
\end{tabular}

Out of 16 MRSA, highest prevalence was obtained from age group of 41-50 years i.e 5(31.3\%). The number of MRCoNS was same in all age groups with prevalence of $50 \%$. There was no significant association $(p=0.25)$ between age group and prevalence of MRSA, MSSA, MRCoNS and MSCoNS (Table 2).

Table 2: Age wise prevalence of MRSA, MSSA, MRCoNS and MSCoNS

\begin{tabular}{|c|c|c|c|c|c|c|}
\hline Age Group & MRSA (\%) & MSSA (\%) & MRCoNS (\%) & MSCoNS (\%) & Totals (\%) & $P$ value \\
\hline $0-10$ & - & $2(8.7)$ & $1(20)$ & - & $3(5.8)$ & \\
\hline $11-20$ & $2(12.5)$ & $1(4.3)$ & - & $1(12.5)$ & $4(7.7)$ & \\
\hline $21-30$ & $1(6.3)$ & $9(39.2)$ & $1(20)$ & - & $11(21.1)$ & \\
\hline $31-40$ & $2(12.5)$ & $5(21.8)$ & - & $4(50)$ & $11(21.1)$ & \\
\hline $41-50$ & $5(31.3)$ & $2(8.7)$ & $1(20)$ & $2(25)$ & $10(19.2)$ & \\
\hline $51-60$ & $3(18.7)$ & $2(8.7)$ & $1(20)$ & - & $6(11.6)$ & \\
\hline $61-70$ & $2(12.5)$ & $1(4.3)$ & - & $1(12.5)$ & $4(7.7)$ & \\
\hline $71-80$ & - & - & - & - & - & \\
\hline $81-90$ & $1(6.3)$ & $1(4.3)$ & $1(20)$ & - & $3(5.8)$ & 0.25 \\
\hline Totals & $16(30.8)$ & $23(44.2)$ & $5(9.6)$ & $8(15.4)$ & $52(100)$ & \\
\hline
\end{tabular}


All MRSA isolates showed resistance towards cefoxitin and penicillin followed by amoxycillin i.e. 93.7\%. In MSSA maximum resistance was shown against erythromycin with $73.9 \%$. MRCoNS showed highest resistance was towards cefoxitin, amoxicillin and penicillin with $100 \%$. MSCoNS shows maximum resistance against penicillin with $75 \%$ (Table 3 ).

Table 3: Antibiogram of methicillin resistant Staphylococcus species

\begin{tabular}{lcccc}
\hline \multicolumn{1}{c}{ Antibiotics } & $\begin{array}{c}\text { MRSA resistant to } \\
\text { antibiotics(\%) }\end{array}$ & $\begin{array}{c}\text { MSSA resistant to } \\
\text { antibiotics(\%) }\end{array}$ & $\begin{array}{c}\text { MRCoNS resistant to } \\
\text { antibiotics(\%) }\end{array}$ & $\begin{array}{c}\text { MSCoNS resistant to } \\
\text { antibiotics(\%) }\end{array}$ \\
\hline Amoxicillin(10mcg) & $15(93.7)$ & $11(47.8)$ & $5(100)$ & $4(50)$ \\
Cefoxitin(30mcg) & $16(100)$ & - & $5(100)$ & - \\
Cefixime(5mcg) & $10(62.5)$ & $8(34.8)$ & $3(60)$ & $3(37.5)$ \\
Ciprofloxacin(5mcg) & $7(43.7)$ & $3(13)$ & $4(80)$ & - \\
Clindamycin(2mcg) & $3(18.7)$ & $4(17.4)$ & $3(60)$ & $3(37.5)$ \\
Cotrimoxazole(25mcg) & $9(56.3)$ & $6(26.1)$ & $2(40)$ & $4(50)$ \\
Coxacillin(5mcg) & $12(75)$ & $5(21.7)$ & $3(60)$ & $3(37.5)$ \\
Erythromycin(15mcg) & $13(81.3)$ & $17(73.9)$ & $4(80)$ & $4(50)$ \\
Gentamycin(10mcg) & $1(6.3)$ & $1(4.3)$ & $4(80)$ & $1(12.5)$ \\
Penicillin(10mcg) & $16(100)$ & $13(56.5)$ & $5(100)$ & $6(75)$ \\
Tetracycline(30mcg) & $5(31.2)$ & $3(13)$ & $3(60)$ & $2(25)$ \\
\hline
\end{tabular}

Out of 39 Staphylococcus aureus, 29(74.4\%) were weak biofilm producers, $7(17.9 \%)$ was moderate biofilm producer and $3(7.7 \%)$ was strong biofilm producer. Among 13 CoNS, 12(92.3\%) was weak and 1(7.7\%) was strong biofilm producer. There was no significant association $(p=0.169)$ between biofilm formation capacity and Staphylococcus species (Table 4).

Table 4: Biofilm production by Staphylococcus species in tissue culture plate method

\begin{tabular}{rcccc}
\hline Types of media & Biofilm formation & S.aureus $(\mathbf{n}=\mathbf{3 9})$ & CoNS $(\mathbf{n}=\mathbf{1 3})$ & P-value \\
\hline \multirow{3}{*}{ TSB $+1 \%$ Glucose } & Weak/Non & $29(74.4 \%)$ & $12(92.3 \%)$ & \\
& Intermediate & $7(17.9 \%)$ & - & 0.169 \\
\hline
\end{tabular}

Biofilm producing Staphylococcus species shows erythromycin with $90.9 \%$ (Table 5). maximum resistance against penicillin and

Table 5: Antibiotic susceptibility pattern of biofilm producing non producing Staphylococcus species (TSB $+1 \%$ Glucose)

\begin{tabular}{lcccc}
\hline \multirow{2}{*}{ Antibiotics } & \multicolumn{2}{c}{ Biofilm producer $(\mathbf{n = 1 1 )}$} & \multicolumn{2}{c}{ Biofilm Non Producer(n=41) } \\
\cline { 2 - 4 } & Resistant(\%) & Sensitive(\%) & Resistant(\%) & Sensitive(\%) \\
\hline Amoxicillin(10mcg) & $8(72.7)$ & $3(27.3)$ & $27(65.8)$ & $14(34.2)$ \\
Cefoxitin(30mcg) & $8(72.7)$ & $3(27.3)$ & $13(31.7)$ & $28(68.3)$ \\
Cefixime(5mcg) & $4(36.4)$ & $7(63.6)$ & $18(43.9)$ & $23(56.1)$ \\
Ciprofloxacin(5mcg) & $6(54.5)$ & $5(45.5)$ & $8(19.5)$ & $33(80.5)$ \\
Clindamycin(2mcg) & $3(27.3)$ & $8(72.7)$ & $10(24.4)$ & $31(75.6)$ \\
Cotrimoxazole(25mcg) & $6(54.5)$ & $5(45.5)$ & $15(36.6)$ & $26(63.4)$ \\
Coxacillin(5mcg) & $8(72.7)$ & $3(27.3)$ & $15(36.6)$ & $26(63.4)$ \\
Erythromycin(15mcg) & $10(90.9)$ & $1(9.1)$ & $28(68.3)$ & $13(31.7)$ \\
Gentamycin(10mcg) & $3(27.3)$ & $8(72.7)$ & $4(9.7)$ & $37(90.3)$ \\
Penicillin(10mcg) & $10(90.9)$ & $1(9.1)$ & $30(73.1)$ & $11(26.9)$ \\
Tetracycline(30mcg) & $4(36.4)$ & $7(63.6)$ & $9(21.9)$ & $32(78.1)$ \\
\hline
\end{tabular}




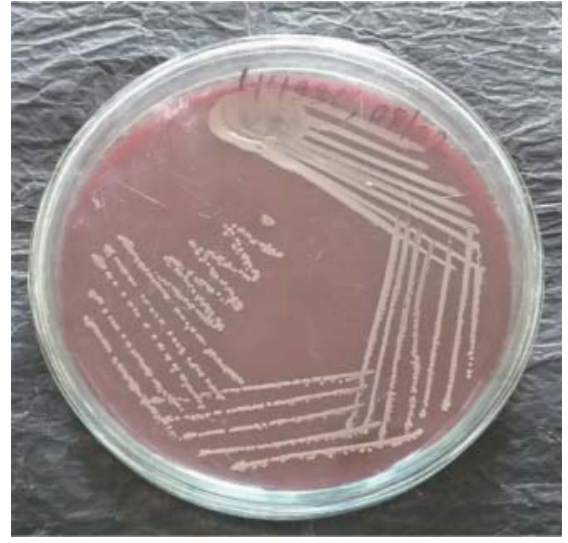

Photograph 1: Culture of Staphylococcus aureus in Blood Agar (pinpoint, smooth, glistening, densely opaque colonies with butyrous consistency)

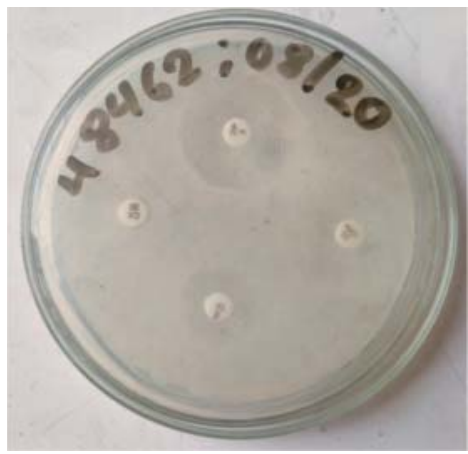

Photograph 3: Antiobiotic susceptibility of S. aureus Sensitive: Ciprofloxacin ; Resistant: Erythromycin, Amoxicillin, Cefoxitin

\section{DISCUSSION}

In this study 60 isolates were found to be Gram positive bacteria out of which 52 (86.67\%) were Staphylococcus species. Among them $39(65 \%)$ were found to be Staphylococcus aureus and 13 (21.67\%) were Coagulase negative Staphylococcus species (CoNS). In the report of Kumari et al. (2008), S. aureus occupied $83.67 \%$ out of total 98 gram positive isolates. A study of Belbase et al. (2017) shows $20.9 \%$ S. aureus out of 364 cultures positive. In our study CoNS was second predominant among Gram positive bacteria with $21.67 \%$. According to Abdel et al. (2018), 52\% were S. aureus and $48 \%$ were CoNS out of 150 isolates of Staphylococcus speciesand in study of Upreti et al. (2018),S. aureus (56.9\%) was common isolate and CoNS (7.8\%) was second predominant bacteria. High frequency might be due

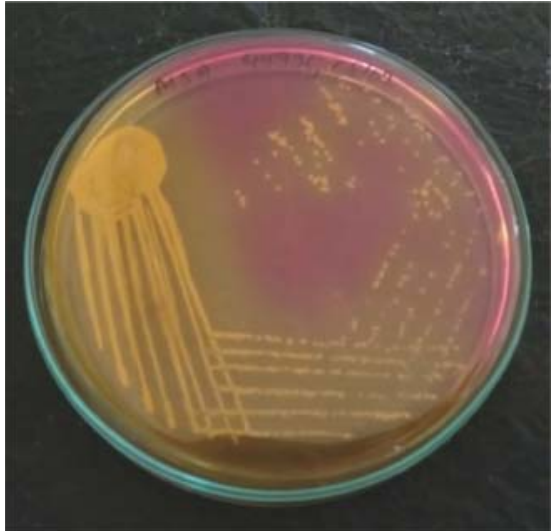

Photograph 2: Culture of Staphylococcus aureus in Mannitol Salt Agar (pinpoint yellowish colonies)

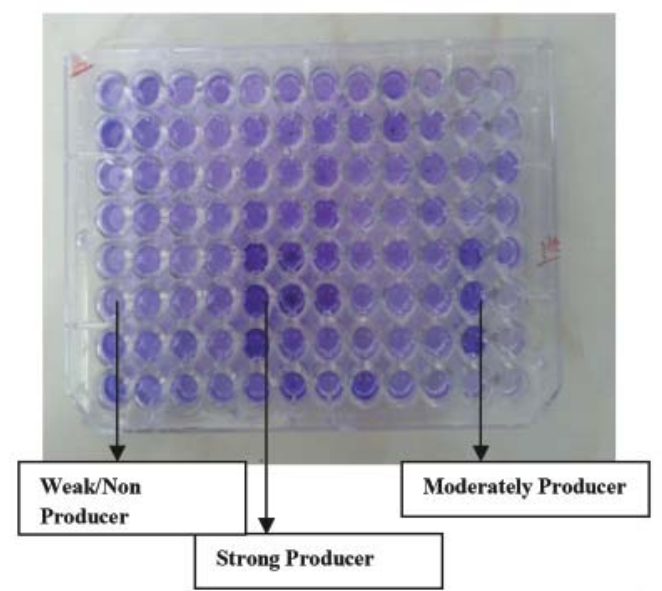

Photograph 4: Biofilm production by Tissue Culture Plate Method

to its ubiquitous nature and large number of virulence factors associated with it.

In this study the prevalence of MRSA was 16(30.8\%), MSSA was 23(44.2\%), MRCoNS was 5(9.6\%) and MSCoNS was $8(15.4 \%)$. The study done in Kathmandu valley by Shrestha et al. (2009) reported $45 \%$ as MRSA from nosocomial $S$. aureus. Study done in Eastern Nepal by Kumari et al. (2008) showed 26.14\% MRSA. Similar study done in western parts of Nepal by Tiwari et al. (2009) also had shown high rate of MRSA isolate (69.1\%). Variations in prevalence of MRSA may be due to infection control measures, antibiotic prophylaxis and treatments used in each ward/hospital and clonal and epidemic nature of microorganisms (Stefani and Varaldo 2003; Robinson and Enright 2004). Likewise 
prevalence of MRCoNS was $9.6 \%$ in our study which was different from the prevalence rate of Maharjan (2017) 28.7\% and Begum et al. (2011) 4\%. In the study conducted by Singh et al. (2016) the prevalence ranges from $48.2 \%$ to $60 \%$ which was higher than our studies. There was statistically no significant association $(p=0.98)$ and $(p=0.29)$ between type of sample and prevalence of MRSA and MSSA and MRCoNS and MSCoNS respectively. In contrast to our study, Mahmood et al. (2010) reported highest prevalence of MRSA from wound swab (35.2\%) and MRCoNS from urine (34\%). Overall data shows lower rate of MRSA and MRCoNS than MSSA and MSCoNS in our study.

In our study the highest prevalence of MRSA was obtained from age group of $41-50$ years with $31.3 \%$. The study of Shahi et al. (2018) observed highest percentage $(47.6 \%)$ of MRSA was isolated from the age group of above 60 years. This might be due to the reduced immune system and use of high dose of medication. There was statistically no significant association $(p=0.25)$ between age group and prevalence of MRSA and MRCoNS.

The antibiotic sensitivity pattern of MRSA showed maximum resistance was towards cefoxitin and penicillin with $100 \%$ followed by amoxycillin i.e. $15(93.7 \%)$. Similar type of result was reported by Tiwari et al. (2009) where all MRSA strains were found resistant to penicillin and $91.9 \%$ were resistant to amoxicillin. The study conducted by Shrestha (2016) and Kumari et al. (2008) also showed higher resistance to amoxicillin with $94.7 \%$ and $91.9 \%$ respectively which resembles to our study. Homogeneous resistance towards betalactams like amoxicillin (93.7\%) and cloxacillin (75\%) resistant MRSA was also observed in our study which is comparable with the study of Shahi et al. (2018). This may be due to presence of intrinsically developed beta-lactamase in MRSA strain. However lower rate of resistance was reported towards gentamycin with $6.3 \%$ in comparison with the study of Belbase et al. (2017) which reported $31.6 \%$ resistance to gentamycin. This may be due to intravenous route of administration and thus a less-commonly used antibiotic that makes abuse difficult (Obiazi et al. 2007). In case of MSSA, maximum resistance was observed against erythromycin with $73.9 \%$ which was higher than previous study done by Sanjana et al. (2010) who reported 58.6\% resistance towards erythromycin. Also MSSA has showed 56.5\% resistivity towards penicillin. This study showed that all MRSA isolates were significantly more resistant to antibiotics and same result was also obtained in MRCoNS.

The antibiotic resistivity pattern of MRCoNS showed maximum resistance was towards cefoxitin, amoxycillin and penicillin with $100 \%$ which is comparable with result of Sharma et al. (2010) with $100 \%$ resistivity towards penicillin group of antibiotics. Similarly $80 \%$ resistance was observed against erythromycin and ciprofloxacin which was higher than Maharjan (2017) who reported resistance rate of erythromycin as $52.2 \%$ and ciprofloxacin as $73.9 \%$. The lower rate of resistance towards erythromycin may be due to extensive use for both serious and minor Staphylococcal infections. The present study also showed that MRCoNS are comparatively more resistant to multiple antimicrobial agents than MSCoNS.

In this study, 52 isolates of Staphylococcus species were tested for biofilm production by Tissue Culture Plate Technique (TCP).Out of 39 Staphylococcus aureus, $29(74.4 \%)$ was found to be weak/non biofilm producer, $7(17.9 \%)$ was found to be moderate biofilm producer and $3(7.7 \%)$ was found to be strong biofilm producer in $\mathrm{TSB}+1 \%$ Glucose media. There was statistically no significant association $(p=0.169)$ between biofilm formation capacity and Staphylococcus species. Our result can be compared with Tuladhar (2018) where $78.4 \%, 12.74 \%$ and $8.8 \%$ were weak/non, moderate and strong biofilm producer respectively. Our result was consistent with another study from Algeria by Lotfi et al. (2014) which showed 8\% strongly adherent, $20 \%$ moderately adherent, $40 \%$ weakly adherent and $32 \%$ non adherent strains. The study by Neopane et al. (2018) reported $34.88 \%$ weak biofilm production, $27.90 \%$ moderate production and $6.97 \%$ strong biofilm production by the TCP method. Likewise among 13 CoNS, $12(92.3 \%)$ was found to be weak/non biofilm producer and $1(7.7 \%)$ was strong biofilm producer and there was no moderate biofilm producer in TSB $+1 \%$ Glucose media. Tuladhar (2018) also reported $81.25 \%$, $16.6 \%, 2.1 \%$ as weak/non, moderate and strong biofilm producer respectively which is slightly similar to our study.

With regards to biofilm producing isolates in TSB $+1 \%$ Glucose media (11), maximum resistance was shown by penicillin and erythromycin with $90.9 \%$. The isolates were highly sensitive to clindamycin and gentamycin 
with $72.7 \%$. The study of Neopane et al. 2018 also showed maximum resistance towards penicillin with $86.7 \%$ and erythromycin with $50 \%$ in biofilm producing S. aureus. In our study, rate of resistance is slightly higher in biofilm producing isolates comparing to other isolates. These results indicate biofilm may be one of the major factors for increasing resistance. Therefore, low-concentration combination therapies can be used to eradicate biofilm-related staphylococcal infections, including those by MRSA (Wu et al. 2013).

\section{CONCLUSION}

Staphylococcus aureus was predominant followed by CoNS among Gram positive organisms and were frequently isolated from blood. The incidence of MRSA was high in age group 41-50. Most of the clinical isolates of methicillin resistant Staphylococcus species were resistance towards $\beta$-lactams like penicillin, amoxicillin, cloxacillin etc, Macrolids, Fluoroquinolones. Resistance is slightly higher in biofilm producing isolates comparing to other isolates.

\section{ACKNOWLEDGEMENTS}

The authors acknowledges profound gratitude to all the staffs and faculties of Department of Microbiology, TriChandra Multiple Campus, Ghantaghar, Kathmandu and all concerned persons at Pathology Department and Lab Technician of Sukraraj Tropical Infectious Disease Hospital and Med-Micro Research Laboratory for their support and guidance at all stages of my work.

Authors are thankful to UGC (University Grants Commission), Nepal for providing financial support.

\section{CONFLICT OF INTEREST}

The authors declare that thay have no conflict of interest.

\section{REFERENCES}

Abdel Halim RM, Kassem NN, and Mahmoud BS (2018) Detection of Biofilm Producing Staphylococci among Different Clinical Isolates and Its Relation to Methicillin Susceptibility. Open Access Maced J Med Sci 6(8): 1335-1341.

Begum ES, Anbumani N, Kalyan J and Malika M (2011) Prevalence and antimicrobial susceptibility pattern of Coagulase negative staphylococci. Int J Med Public Health 1(4): 59-62.

Belbase A, Pant ND, Nepal K, Neupane B, Baidya, R and Lekhak, B (2017) Antibiotic resistance and biofilm production among the strains of Staphylococcus aureus isolated from pus/wound swab samples in a tertiary care hospital in Nepal. Ann Clin Microbial Antimicrob 16(1): 15.

Clinical and Laboratory Standards Institute (CLSI) (2019) Performance Standards for Antimicrobial Susceptibility Testing, USA: CLSI: M100- S29. Wayne, PA. USA.

Donlan RM (2002) Biofilms: microbial life on surfaces. Emerging Infectious Diseases 8(9): 881890.

Donlan RM and Costerton W (2002) Biofilms: Survival mechanisms of clinically relevant Microorganisms. Clin Microbiol Rev 15(2):167-193.

Gotz F (2002) Staphylococcus and biofilms. Mol. Microbiol 43: 1367-1378.

Jain A and Agarwal A (2009) Biofilm production: a marker of pathogenic potential of colonizing and commensal Staphylococci. J. Microbiol Methods 76: 88-92.

Kumari N, Mohapatra TM and Singh YI (2008) Prevalence of Methicillin Resistant Staphylococcus aureus (MRSA) in a Tertiary Care Hospital in Eastern Nepal. J Nepal Med Assoc 47: 53-56.

Lotfi G, Hassaine H, Klouche N, Khadir A, Aissaoui N, Rabehi FNE and Zingg W (2014)Detection of biofilm formation of a collection of fifty strains of Staphylococcus aureus isolated in Algeria at the University Hospital of Tlemcen. JBR 6: 1-6.

Maharjan P (2017) Coagulase negative Staphylococci from different Clinical samples and their antibiotic susceptibility pattern. M.Sc. Dissertation submitted to Tri-Chandra Multiple Campus.

Mahmood K, Tahir T, Jameel T, Ziauddin A and Aslam HF (2010) Incidence of Methicillin-resistant Staphylococcus aureus (MRSA) causing nosocomial Infection in a Tertiary Care Hospital. Annals KEMC 16: 2.

Neopane P, Nepal HP, Shrestha R, Uehara O and Abiko Y (2018) In vitro biofilm formation by Staphylococcus aureus isolated from wounds of hospital-admitted patients and their association with antimicrobial resistance. Int J Gen Med 11: 25-32.

Obiazi H, Nmorsi O, Ekundayo A and Ukwandu NCD 
(2007) Prevalence and antibiotic susceptibility pattern of Staphylococcus aureus from clinical isolates grown at 37 and 44 degrees $C$ from Irrua, Nigeria. AFR J Microbiol Res 1: 57-60.

Reid G (1999) Biofilms in infectious disease and on medical devices. Int J Antimic Ag 11:223-6.

Robinson DA and Enright MC (2004) Multilocus sequence typing and the evolution of methicillinresistant Staphylococcus aureus. Clin Microbiol Infect 10(2): 92-7.

Sanjana RK, Shah R, Chaudhary N and Singh YI (2010) Prevalence and antimicrobial susceptibility pattern of methicillin resistant Staphylococcus aureus in CMS -teaching hospital: Premilinary report. JCMS-Nepal 6(1):1-6.

Shahi K, Rijal K, Adhikari N, Shrestha U, Banjara M, Sharma V and Ghimire, P (2018) Methicillin Resistant Staphylococcus aureus: Prevalence and Antibiogram in Various Clinical Specimens at Alka Hospital. Tribhuvan University Journal of Microbiology 5: 77-82.

Sharma V, Jjindal N and Devi P (2010) Prevalence of methicillin resistance coagulase negative Staphylococci in a tertiary care hospital. Iran J Microbiol 2(4): 185-188.

Shrestha (2016) Detection of biofilm formation and their correlation with methicillin resistance Staphylococcus aureus isolated from Clinical specimens. Dissertation submitted to Department of microbiology, Kantipur College of Medical Science, Sitapaila, Kathmandu.

Shrestha B, Pokhrel B and Mohapatra T (2009) Study of nosocomial isolates of Staphylococcus aureus in tertiary care hospital in Nepal. Nepal Med Coll Journal 11(2):123-126.

Singh S, Dhawan B, Kapil A, Kabra SK, Suri A, Sreenivas V and Das BK (2016) Coagulase negative Staphylococci causing blood stream infection at an Indian Tertiary care hospital: Prevalence, antimicrobial resistance and molecularcharacterization. Indian J Med Microbial 34: 500-505.

Stefani S and Varaldo PE (2003) Epidemiology of methicillinresistant Staphylococci in Europe. Clin Microbiol Infect 9(12): 1179-86.

Stepanovic S, Vukovi D, Hola V Di Bonaventura G, Djukic S, Cirkovic I and Ruzicka F (2007) Quantification of biofilm in microtiter plates: overview of testing conditions and practical recommendations for assessment of biofilm production by Staphylococci. APMIS 115(8): 891899.

Tiwari HK, Das AK, Sapkota D , Sivrajan KK and Pahwa VK (2009) Methicillin resistant Staphylococcus aureus: prevalence and antibiogram in a tertiary care hospital in western. Nepal J Infect Dev Ctries 3(9): 681-4.

Tuladhar RS (2018) Antibiotic Susceptibility Pattern of biofilm producing Staphylococci isolated from different clinical specimens. M.Sc. Dissertation submitted to Tri-Chandra Multiple Campus.

Upreti N, Rayamajhee B, Sherchan SP, Choudhari MK and Banjara MR (2018) Prevalence of methicillin resistant Staphylococcus aureus, multidrug resistant and extended spectrum $\beta$-lactamase producing gram negative bacilli causing wound infections at a tertiary care hospital of Nepal. Antimicrob Resist Infect Control 7: 121.

Wimpenny J, Manz W and Szewzyk U (2000) Heterogeneity in biofilms. FEMS Microbiol Rev 24: 661-671.

Wu WS, Chen CC, Chuang YC, Su BA, Chiu YH, Hsu HJ, Ko WC and Tang HJ (2013) Efficacy of combination oral antimicrobial agents against biofilm-embedded methicillin-resistant Staphylococcus aureus. J Microbiol Immunol Infect 46(2):89-95. 